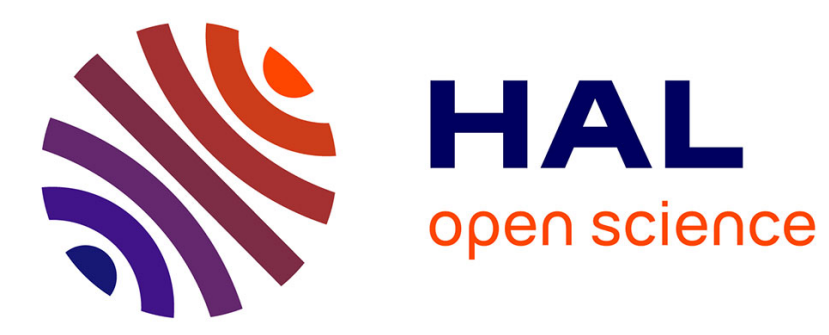

\title{
LASERS IN CROSSED BEAM EXPERIMENTS
}

J. Baudon

\section{To cite this version:}

J. Baudon. LASERS IN CROSSED BEAM EXPERIMENTS. Journal de Physique Colloques, 1985, 46 (C1), pp.C1-25-C1-36. 10.1051/jphyscol:1985103 . jpa-00224472

\section{HAL Id: jpa-00224472 https://hal.science/jpa-00224472}

Submitted on 1 Jan 1985

HAL is a multi-disciplinary open access archive for the deposit and dissemination of scientific research documents, whether they are published or not. The documents may come from teaching and research institutions in France or abroad, or from public or private research centers.
L'archive ouverte pluridisciplinaire HAL, est destinée au dépôt et à la diffusion de documents scientifiques de niveau recherche, publiés ou non, émanant des établissements d'enseignement et de recherche français ou étrangers, des laboratoires publics ou privés. 


\title{
LASERS IN CROSSED BEAM EXPERIMENTS
}

\author{
J. Baudon \\ Laboratoire de Physique des Lasers, Université Paris-Nord, \\ Avenue J.B. Clément, 93430 Villetaneuse, France
}

Résumé

On examine les diverses utilisations possibles du laser dans des expériences de collision en jets croisés. L'exposé est limité aux collisions de faible énergie (quelques dizaines de meV) entre atomes excités et atomes ou molécules. Le premier rôle que peut jouer le laser dans ces expériences est celui de sélecteur, permettant aussi bièn la préparation de I'état initial que l'analyse de l'êtat final, qu'il s'agisse d'un niveau atomique bien défini (radiatif ou métastable), d'une classe de vitesse, d'un sous-niveau Zeeman. Dans ces applications, le laser n'agit par sur la collision elle même. Il n'en va plus de même aux puissances élevées et à des fréquences dẻcalêes par rapport aux fréquences atomiques. Quelques suggestions sont faites sur les possibilités d'étudier, en jets croisés, ces collisions assistées par laser.

\section{Abstract}

Different possible uses of lasers in crossed beam experiments are examined. The scope is limited to low energy collisions ( $f$ ew tens of meV) between excited atoms and atoms or molecules. The first role of the laser in this kind of experiments is that of a selector, allowing either the preparation of the incoming channel or the analysis of the outcoming one, by selecting one definite atomic level iradiative or metastable), one class of velocities, one magnetic sublevel. In all these applications, the laser does not act on the collision process itself. The situation is quite different at high laser powers and frequencies detuned with respect to atomic frequencies. Some suggestions are made about the feasability of crossed beam studies of these laser-assisted collisions.

The goal in crossed beam experiments is to prepare as well as possible the initial momenta of the collision partners, or equivalently, the relative velocity $v_{r}$ and the initial direction in the center-of-mass frame $\left(\Theta_{\mathrm{CM}}=0\right)$. According to the species under investigation, and to the financial support, one may use either monokinetic (supersonic) beams, or thermal beams plus velocity selector, or pulsed thermal beams plus a time-of-flight analysis. The collision energy is varied either by heating or cooling, or by selecting variable times of flight, or by changing the relative angle between the two beams. In this type of experiments the laser can be used in two different ways : (i) as a tool to complete and improve the preparation or the analysis of the collision partners, with no appreciable effect on the collision itself : (ii) as a mean to change the nature of the collision process.

I - LASERS AS SELECTORS

Except for ground state species, the internal state of the collision partners before and after the collision is generally not controlled in simple crossed beam experiments. The first possible role of the laser is to act as a selector, or an 
analyzer, of states and eventually also of the velocites. These uses of laser light are summarized in fig. 1 .

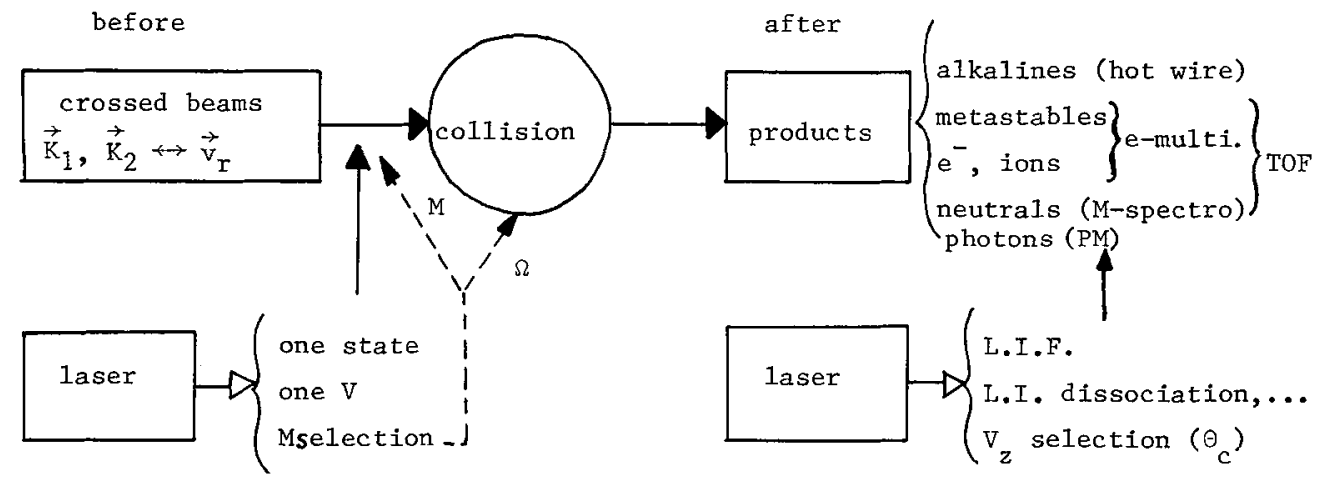

Fig. 1 - Lasers as selectors

The laser can be used to produce and select radiative states-the laser beam is sent directly into the collision volume -, or to depopulate selectively long 1ived (e.g. metastable) levels before the collision volume. These selection properties can obviously be used as well in the analysis of the final state (cf. for instance the laser induced fluorescence method). Good optical pumping conditions generally require :

(i) a continuous tunable laser locked on an atomic line,

(ii) a sufficently high power, to get a stationnary pumping regime, but, in some cases, not too high, to avoid undesirable saturation effects,

(iii) a convenient choice of the excitation scheme.

\section{I.1 - Population, or depopulation, of a specific state}

a) Radiative levels of alkali atoms (or alkaline-earths) are populated directly from ground states. Many experiments of this kind have been performed to study excited atom or ground state atom collisions at thermal energies. We shal1 come back to this case when considexing the M-selection. Other experiments have been devoted to excited alkali-on-molecule collisions, for instance :

- fine structure transition in $\mathrm{K}^{*}(4 \mathrm{P})$, or $\mathrm{Rb}^{*}(5 \mathrm{P})+\mathrm{H}_{2}$ or $\mathrm{D}_{2}$ collisions [1]. One originality of this experiment consists in that the rotational temperature is varied by seeding the $\mathrm{H}_{2}\left(\mathrm{D}_{2}\right)$ supersonic beam with heavier atoms, such as argon ( $\mathrm{T}_{\text {rot }}$ is estimated from the energy balance between the different degrees of freedom). 
- energy transfer (electronic to vibrational) in $\mathrm{Na}(3 \mathrm{p}$ ) on triatomic molecule $\left(\mathrm{CO}_{2}, \mathrm{~N}_{2} \mathrm{O}\right)$ collision [2]; the final relative energy $\mathrm{E}^{\prime}{ }_{\mathrm{CM}}$ is obtained at each scattering angle by means of a velocity selector set in front of the alkali-atom detector, and the doubly-differentia1 cross section $\mathrm{d}^{2} \sigma /\left(\mathrm{dE}^{\prime} \mathrm{CM}^{\cdot} \cdot \mathrm{d}_{\mathrm{CM}}\right)$ is measured.

- reactive scattering experiments, using a "simple" (but differential) mass analysis, e.g. for the reactions :

$$
\mathrm{Na}(3 \mathrm{~s} \text { or } 3 \mathrm{p})+\mathrm{HCl} \rightarrow \mathrm{NaCl}+\mathrm{H}
$$

(Here no information is obtained about the internal state of the product), or the laser induced fluorescence method, as in :

$$
F+I X \rightarrow I F(v)+X\left({ }^{2} p_{3 / 2,1 / 2}\right) \text {, where } X \text { is halogen [4]; }
$$

here relative total cross sections are obtained for each value of $v$, in the range $0-16$. The same method has been used for the reactions : $\mathrm{Cs}^{*}\left(7 \mathrm{P}_{3 / 2,1 / 2}\right)+\mathrm{H}_{2} \rightarrow \mathrm{CsH}+\mathrm{H}[5]$; a first laser is used to populate $\mathrm{Cs}^{*}$ and a second one analyses the internal state of product.

- collision between two excited alkali-atoms [6].

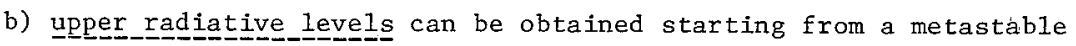
level. This is typically the case for rare gas atoms. For instance, the HornbeckMolnar process :

$$
\mathrm{He} *(5 \mathrm{P})+\mathrm{He}\left({ }^{1} \mathrm{~S}_{\mathrm{O}}\right) \rightarrow \mathrm{He}_{2}^{+}+\mathrm{e}
$$

has been recently studied [7]. Excited helium atoms are produced within a metastable $\left(2^{3} \mathrm{~S}\right) \mathrm{He}$ beam by a frequency doubled $\mathrm{CW}$ dye laser ; about $10 \mathrm{~mW}$ are obtained at $\lambda=294.5 \mathrm{~nm}$, by using an intra-cavity ADA doubler crystal.

of course upper levels (e.g. Rydberg states) can be also populated from a radiative leve1, by means of a second laser beam.

c) A specific metastable state can be depopulated before the collision volume and also eventually before the metastable atom detector. This method has been used mainly for rare gas atoms [8]. For practical reasons (spectral range), the neon atom is here the ideal one. For example, very detailed studies of $\mathrm{Ne}{ }^{*}\left(3 s,{ }^{3} \mathrm{P}_{0}\right)-\mathrm{Ne}$ and $\mathrm{Ne}^{\star}\left(3 \mathrm{~s}, \mathrm{P}_{2}\right)$ - Ne collisions have been performed [9]. It is noteworthy that the laser selector is isotope selective; this gives a very easy way to separe the exchange process from the direct one, which helps to clarify the experimental test on the potential energy curves, especially in cases where many molecular states are involved (e.g. for the ${ }^{3} \mathrm{P}_{2}$ level) [10]. 


\section{I.2 - Velocity selection}

When the atomic beam is well-collimated but has a wide velocity distribution (thermal beam), the Doppler effect allows to pump selectively a narrow band of velocity. This technique is particulary useful when the second beam is monokinetic.

For radiative states (alkali atoms) this laser velocity selection provides a purely optical method to measure elastic or inelastic differential cross sections [11] (see fig. 2) : a first collinear laser beam $\left(\omega_{1}\right)$ selects one velocity $\vec{v}_{A}$ within the excited atom beam; if the initial velocity $\vec{v}_{B}$ of the second partner is unique, the initial relative velocity $\vec{v}_{r}$ is then well defined; a second laser beam $\left(\omega_{2}\right)$, inducing a transition from the excited state $\left(e_{1}\right)$ of A to an upper excited state ( $e_{2}$ ), and collinear to $\vec{v}_{r}$, selects a definite value of the projection of $\vec{v}_{r}$ of the final velocity, i.e. a definite value of the $\mathrm{CM}$ scattering angle. An inelastic collision can be studied as well by tuning the second lasez on a transition starting from another state $\left(\mathrm{e}^{\prime}\right)$ of $A$ after the collision. The observation is made via the fluorescence $\left(\omega_{f}\right)$ from $\left(e_{2}\right)$.
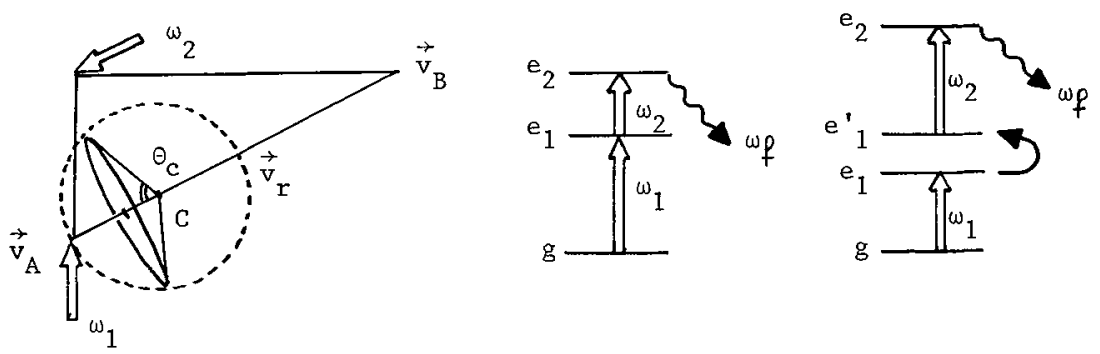

Fig 2 - Optica1 measurements $s$ of a D.C.S

A velocity selection can be achieved also for a metastable level, by depopulating a narrow velocity band. Fig. 3 shows the time-of-flight distribution of a metastable $\left(3 \mathrm{~s},{ }^{3} \mathrm{P}_{0,2}\right) \mathrm{Ne}$ beam. A laser, tuned on the $1 \mathrm{~s}_{5}{ }^{3} \mathrm{P}_{2}-2 \mathrm{p}_{6}$ transition $(\lambda=614.3 \mathrm{~nm})$ and then conveniently shifted, burns a hole in the distribution, specifically for ${ }^{3} \mathrm{P}_{2}$ level and ${ }^{20} \mathrm{Ne}$ isotope [11]. Owing to the long aquisition times (hours) required in differential measurements, the laser frequency needs to be carefully stabilized. In the present case, a CW dye laser is locked on a saturated absorption Iine shifted by a magnetic field [12]. Up to now, the resolution of this "negative" velocity selector is essentially limited by the angular aperture of the atomic beam ( $\Delta v \approx 80$ to $100 \mathrm{~m} / \mathrm{s}$ ). 


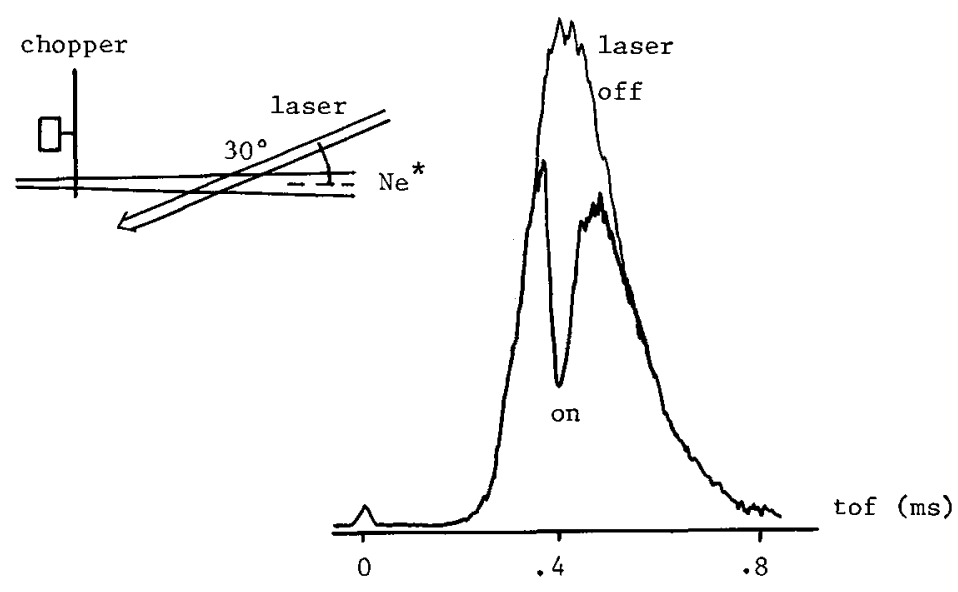

Fig 3 - Laser velocity selection in a Ne* metastable beam.

I.3 - Magnetic sublevel selection

Up to now it has been implicitly supposed that no polarization effect occurs. Notice that this is not necessarily a "natural" case, because of the strong polarization of the laser light, and one has sometimes to take care to eliminate polarization effects. On another hand, a magnetic sublevel selection ( $M$ - selection) is very useful, at least when it is completely controlled.

In low energy collisions rather complex processes may occur at large internuclear distances $\left(R \geqslant 10 a_{0}\right)$ : coupling terms, negligible at small $R$, such as coupling due to the rotation of the molecular axis $\vec{R}$, spin-orbit coupling, etc..., become more and more efficient at large $R$. The problem here comes from the fact that these terms may have comparable magnitudes, which makes difficult to construct the "good" molecular basis. The M - selection of initial and final states offers an interesting way to investigate experimentally these intricate long range effects.

To understand how the laser M - selection operates, one has to consider with attention the reference quantization axis. For sake of clarify we shall consider molecular states dissociating at infinite $R$ into $A^{*}\left({ }^{1} P\right)+B\left({ }^{1} S\right.$ ) (see fig. 4).

When $A B$ is an heteronuclear system, three molecular states have this dissociation limit : $\pi_{+}, \pi_{-}, \Sigma$, for which the componant on $\hat{R}$ of the electronic angular momentum is $+1,-1,0$ respectively. Three quantization axis need to be considered, namely $: \hat{z}_{i}$ for incoming state, $\hat{R}$ for the collision itself and $\hat{z}_{f}$ for the outcoming state. A pure state prepared along $\hat{z}_{i}\left(\right.$ e.g. $\left.M\left(z_{i}\right)=+1\right)$ is generally a coherent superposition of states $| \pm 1>| 0>$,, along $\hat{R}$. The outcoming combination along $\hat{R}$ is different from the incoming one, $i . e$ it is not globally shifted, because the potentials are different for $\Lambda=1$ and $\Lambda=0$ and also because long range static or dynamical (according to the chosen molecular basis) couplings occur. Finally one gets a definite superposition of outcoming amplitudes $f_{0}, f_{ \pm l}$, refered to $\hat{z}_{f}$. A similar conclusion holds when fine structure, and hyperfine structure exist. 


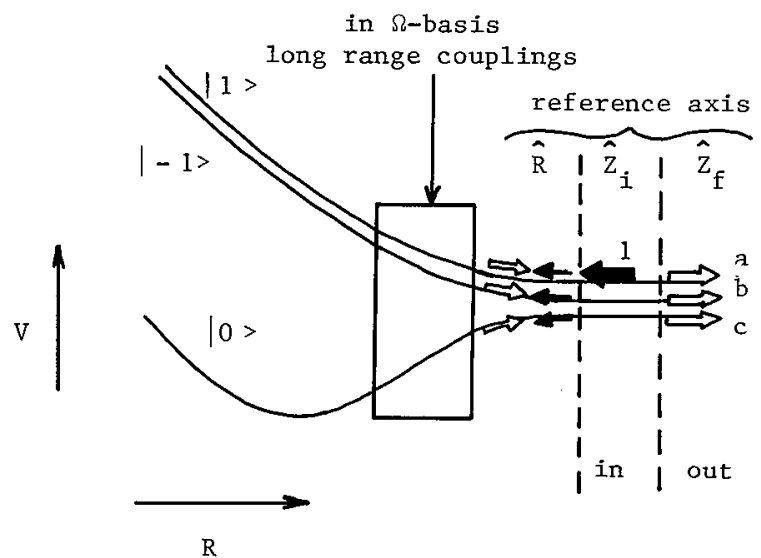

Fig $4-$ M-selection in a $A\left({ }^{1} P\right)+B\left({ }^{1} S\right)$ collision

a) examples for radiative levels

- By choosing different excitation schemes (directions of atom and laser beams, polarization, external magnetic field) it is possible to study a given process (elastic or inelastic) with different superpositions of incoming channels. The relative contributions of different incoming molecular states (and consequently of different types of coupling) to the apparent total cross section for the fine structure transition :

$$
\mathrm{K}\left(4 \mathrm{P}_{3 / 2}\right)+\text { rare gas } \mathrm{X} \rightarrow \mathrm{K}\left(4 \mathrm{P}_{1 / 2}\right)+\mathrm{X}
$$

have been stuđied by this way [13], choosing different excitation schemes, such as : (i) a wide-band laser $(0,1 \AA)$ linearly polarized, with a magnetic field perpendicular to the collision plane, (ii) a narrow-band laser ( $\leq 1 \mathrm{MHz}$ ) circulary polarized. This a1lows a measurement of two quantities :

$$
\mathrm{A}(1 / 2)=\sum_{\mathrm{m}} \sigma(3 / 2,1 / 2 \rightarrow 1 / 2, \mathrm{~m}) ; \mathrm{A}(3 / 2)=\sum_{\mathrm{m}} \sigma(3 / 2,3 / 2 \rightarrow 1 / 2, \mathrm{~m})
$$

as functions of energy $(0.07$ to $0.4 \mathrm{eV})$, from which the relative contributions of states $A^{2} \pi_{3 / 2}$ and $B^{2} \Sigma_{1 / 2}$ are derived.

- From the differential view point, a spectacular effect of M-selection has been found by Düren et al [14] : when excited potassium atoms $K^{*}\left(4^{2} \mathrm{P}\right)$ are prepared by a $\sigma^{+}$laser light ( refered to $\hat{z}_{i}$ perpendicular to the initial velocity plane) and then collide with e.g. $K_{r}$ atoms, a right-left assymetry is observed in the differential cross section, and the difference between $+\theta$ and $-\theta$ scattered intensities oscillate with $\Theta$. 
- In atom-diatom collisions, the initial angular momentum of the excited atom, and a part of the relative angular momentum can be transfered to the molecule. A M-selection with respect to the quantization axis $\hat{z}_{i}$ perpendicular to the collision plane, well defined in a differential experiment, allows a direct study of these angular momentum transfers, for instance in $\mathrm{Na} *(3 \mathrm{P})+\mathrm{N}_{2}$ or $\mathrm{H}_{2}$ collisions [15].

b) examp1es_for metastable 1 e_leve1s

- A laser M-selection is possible also with metastable levels by a suitable choice of the optical transition, polarization and external magnetic field. For instance, for $\mathrm{Ne}^{*}\left({ }^{3} \mathrm{p}_{2}\right)$, one can use the Zeeman splittings between $3 \mathrm{~s}^{3} \mathrm{P}_{2}-3_{\mathrm{p}} \mathrm{p}_{2}$ lines to separate different values of $\mathrm{M}_{\mathrm{J}}$.

This method has been applied recently by $\mathrm{H}$. Haberland et al [09] to Ne* - Ne collisions. An important advantage of metastable atoms is that this preparation is made before the collision volume. Therefore it is possible, by means of an appropriate magnetic field $(\vec{B})$ configuration, to rotate adiabatically the quantization axis, for instance from $\hat{z}_{i}$ to the initial direction $\hat{R}_{i}$ of the molecular axis ; "adiabatically" means here that the time evolution of $\vec{B}$ seen by the moving atom is sllow with respect to the precession period, so that the projection of the angular momentum along $\vec{B}$ is a constant. The same technique may be used to tranform adiabatically. $\hat{R}_{f}$ Into $\hat{z}_{f}$. Finally preparation and analysis are refered to the molecular axis, which gives a very direct view of the rotational coupling effects : for an infiniteiy slow relative motion, the electronic angular momentum would be "attached" to $\hat{R}$ and a pure M-state would be observe along $\hat{R}_{f}$; in fact the rotation of $\hat{R}_{\text {is }}$ not adiabatic and a combination of final M-states is observed. It may be noticed that such a measurement is extremely sensitive : strong depolarizing effects are observed even for very small couplings; hence they do not necessarily imply that molecular potentials obtained via non M-selective measurements are wrong.

- Another way to achieve the M-selection for metastable atoms consists in using the Doppler velocity selection described in paragraph I.2. If the optical pumping takes place within a magnetic field, with a fixed laser frequency, each Zeeman component gives a hole in the velocity distribution, at a definite value of the velocity. Fig. 5 shows the hole profile obtained in neon, with a $\pi$ polarization with respect to the magnetic field $(B \approx 480 \mathrm{G})$; the angle between Ne* beam and laser beam is $30^{\circ}$; the solid lines correspond to the theoretical Zeeman spectrum. For a demonstrative purpose the experimental conditions have been arranged in such a way that the whole spectrum lies within the velocity distribution. Obviously it is preferable to increase the magnitude of $B$ so that just one component remains in the distribution. 

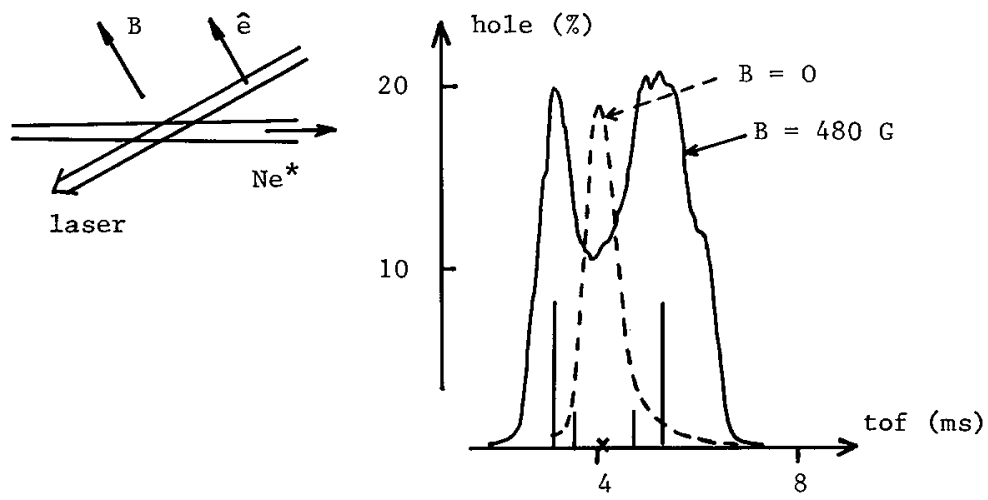

Fig 5 - Laser M-selection in a $\mathrm{Ne}^{*}$ metastable beam

\section{II - LASER ASSISTED COLLISIONS}

When the collision volume is irradiated by a high power laser beam, which frequency is more or less detuned with respect to atomic frequencies, the collision process is no longer independent of the interaction with the electromagnetic (EM) field. As shown in fig. 6 the "main" laser, characterized by its power (or electric field amplitude $\varepsilon_{0}$ ) and detuning $(\delta)$, acts during the collision itse1f. As indicated another laser can be used as a selector.

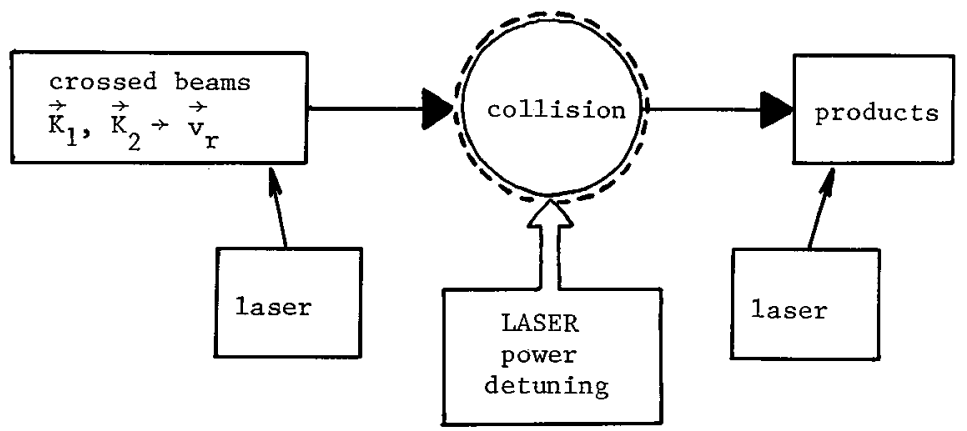

Fig 6 - Laser-assisted collision

To get a correct formulation of the problem, one has to consider as a whole both collision partners, A,B interacting by $V$, the EM field and the interaction $v$ between $A($ or $B)$ and the EM field, i.e. to consider the hamiltonian :

$$
H=h_{A}+h_{B}+h_{R}+V+v
$$


$h_{A, B}$ are the internal hamiltonians of $A, B, h_{R}$ is that of the EM field. At given collision energy and impact parameter the characteristic time for $\mathrm{V}$ is the collision time $\tau_{c}$. The characteristic time for $v$ is $\Omega^{\prime^{-1}}$, where $\Omega^{\prime}$ is the Rabi frequency :

$$
\Omega^{\prime}=\left[\left(\frac{\mu \mathscr{G}_{0}}{\not h}\right)^{2}+\delta^{2}\right]^{1 / 2}
$$

The hamiltonian $h_{A \text { (or } B)}+h_{R}+v$ is that of the dressed atom, whereas in the Born-Oppenheimer approximation, $h_{A}+h_{B}+V$ describes the quasimolecule $A B$. The main theoretical problem here is to establish some preeminence among the various terms contained in $\mathrm{V}$, and $\mathrm{v}$, in other words to construct what we might call "dressed hund's cases". Obviously the problem becomes serious - and interesting - for laser powers large enough to give $\Omega^{\prime} \sim \tau^{-1}$.

Let us turn now to the experiment view point, and more specifically to crossed beam experiments. One knowns the difficulties encountered in studying laser-assisted collisions in cellexperiments, and the high powers genrally required to attain the high intensity limit. Therefore to imagine crossed beam experiments in this field might seem rather utopic since collisional events in this type of experiment come from rare encounters between atoms belonging to two density media. However crossed beams are not necessarily a pure inconvenience : state-selections which are possible in such experiments can allow a separation of the investigated process from many other unuseful ones. Nevertheless one has to choose carefully the studied case and to respect some general requirements :

(i) use low energies (i.e. large collision times and large distances)

(ii) use excited species (potential curves are close, coupling more efficient), with long lifetimes.

(iii) study long-range inelastic processes, involving dipole transitions,

(iv) choose a laser assisted process easy to be characterized,

(v) use a sufficiently high intensity to get $\Omega^{\prime} \geqslant \tau_{c}^{-1}$

A sensitive method to detect even small collisional laser effects consists in using interferences between coherent scattering amplitudes. As an example, let us consider a collision between two equal $\mathrm{Z}$ atoms $\mathrm{A}, \mathrm{A}^{\prime}$; $\mathrm{A}^{\prime}$ is initially in its groundstate (0) whereas $A$ is in a long-lived excited state (1) (metastable or Rydberg). The laser frequency is slightly detuned with respect to an allowed transition, from state (1) to an upper excited state (2), the lifetime of which is large compared to $\Omega^{,-1}$ and to the transit time $\mathrm{T}$ of $\mathrm{A}$ across the laser beam.

If the laser intensity is low $\left(\Omega^{\prime-1}\right.$ is much larger than the duration $\tau_{c}$ of a collision), the collision process is completely decoupled from the laser interaction: a linear time dependent superposition of states (1) and (2) evolves within the laser beam up to a time $t_{c}^{-}$"just" before the collision time $t_{c}$ (but in fact $\simeq-\infty$ for the collision itself). In the time interval $t_{c}^{-}, t_{c}^{+}$(just after collision), the adequate description is the molecular one. The evolution of the system is governed by potentials $1_{u, g}, 2_{u, g}$ (see fig. 7) and the laser has no appreciable effect during that time. 
The outcoming collisional state is then a linear combination of atomic states $A_{0}^{\prime}$, $A_{1,2}$ and $A_{1,2}$, A (quasi-resonant excitation transfer). As the laser induces transition 1-2 for $A$ only, the final $(t=T)$ population in $A_{1}$ takes the following from :

$$
\left|a_{1}(T)\right|^{2}=C[1+\rho \cos (2 \Omega \top T+\emptyset)]
$$

where real quantities $C, \rho$ and $\emptyset$ depend on moduli and relative phaseshift of the direct scattering amplitudes :

$$
\frac{1}{2}\left[f_{g}^{(1)}+f_{u}^{(1)}\right], \frac{1}{2}\left[f_{g}^{(2)}+f_{u}^{(2)}\right]
$$

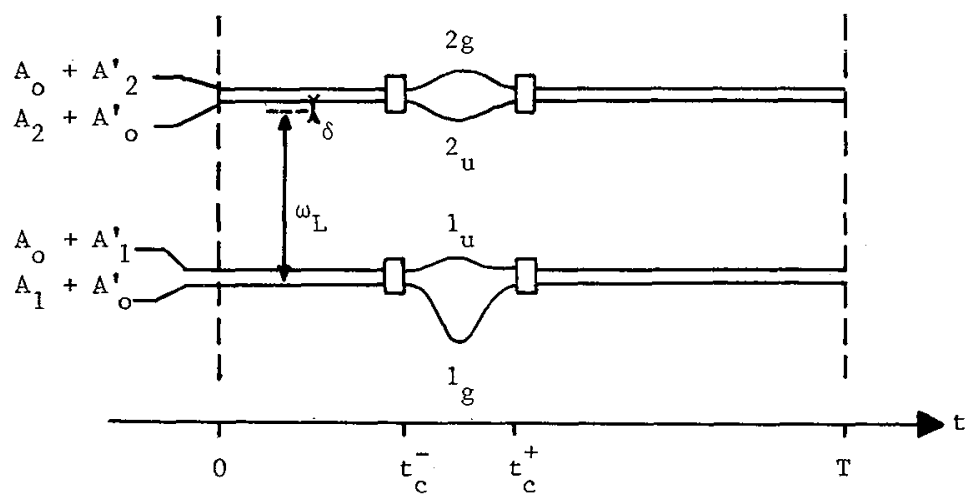

Fig 7 - Symmetric collision in a laser beam

At high laser intensity $\left(\tau_{c} \simeq \Omega^{-1}\right)$, the laser effect can no longer be neglected within the ime internal $t_{c}^{-}, t_{c}^{+}$, for atoms $A$ and for atoms $A^{\prime}$. One is dealing with a 4 -state problem (4 standard molecular states $I_{g}, 2_{u} ; 1_{u}, 2_{g}$, coupled by the radiation field, or 4 dressed molecular states). The final result on $\left|a_{1}(T)\right|^{2}$ consists in an additional interference between direct and exchange scattering amplitudes.

Another example of a laser-modified interference effect is given by an asymmetric collision $A+B$, in which one of the two atoms has an internal angular momentum, for instance: $A\left(1,{ }^{1} S\right)+B\left({ }^{1} S\right)$, combined with $A\left(2,{ }^{1} P\right)+B\left({ }^{1} S\right)$, by means of a laser slighty detuned with respect to the $1,1_{S}-2, I_{P}$ transition. At low laser intensity and for a linear polarization $\hat{e}$ parallel to the initial direction $\hat{R}_{i}$ of the internuclear axis, the incoming collisional state $\left(t=t_{c}^{-}\right)$is a linear combination of states $A\left(1,{ }^{1} S\right), B\left({ }^{1} S\right)$ and $A\left(2,{ }^{1} P_{0}\right), B\left({ }^{1} S\right)$. From the molecular viewpoint; the atomic groundstate is connected to a $\Sigma$ state whereas the excited one is connected to $\Sigma\left({ }^{1} P_{0}\right)$ 
and $\Pi\left({ }^{1} P_{ \pm 1}\right)$ states. Therefore, if a long-range $I-\Sigma$ rotational coupling is present, the outcoming $\left(t=t_{c}^{+}\right)$collisional state takes the form :

$a_{1}\left(t_{c}\right) f_{1}\left|{ }^{1} S\right\rangle+a_{2}\left(t_{c}\right) \sum_{\lambda=0, \pm 1} f_{2}^{(\lambda)}\left|{ }^{1} P_{\lambda}\right\rangle$, where the $f-s$ are scattering amp1itudes. For small scattering angles, the laser acts after the collision $\left(t_{c}{ }^{+} \leqq i t ~ T\right)$ only on the projection : $a_{1}\left(t_{c}\right) f_{1}\left|{ }^{1} S>+a_{2}\left(t_{c}\right) f_{2}^{(0)}\right|_{P_{0}}>$. (at large scattering angles, because of the rotation of $\hat{R}$, any component ${ }^{1} P_{\lambda}$ would have a projection onto $M=0$ along $\hat{e})$. Consequently the final groundstate population contains an interference term depending on $\left|f_{1}\right|,\left|f_{2}^{(0)}\right|$ and their relative phaseshift. As before, this interference term will be modified at large intensities, for which the laser acts on the system during the collision itself.

To conclude about the feasability of such assisted crossed beam experiments, let us point out a promizing experimental method developped recently by Pillet et al [16]. The basic idea is to use transitions between high $\mathrm{n}(\mathrm{n} \simeq 20)$ Rydberg levels, e.g. of $\mathrm{Na}$, induced by a microwave field; for instance :

$$
\mathrm{Na}(\mathrm{ns})+(\mathrm{Na}(\mathrm{ns}) \rightarrow \mathrm{Na}(\mathrm{np})+\mathrm{Na}((\mathrm{n}-1) \mathrm{p})+\mathrm{mh} \nu
$$

Because of the very large values taken by the dipole moments, the so-called highintensity regime is accessible with quite reasonable microwave powers (few $\mathrm{W} / \mathrm{cm}^{2}$ ); the long life time of such states is also an abvious advantage of the method.

\section{REFERENCES}

[1] J. Cuvellier, J.M. Mestdagh, M. Ferray, P. de Pujo and J. Berlande, XIII th ICPEAC, Berlin 1983, p. 603.

[2] G. Jamieson, C.P. Schulz, H.U. Tittes, IV Hertel, XIII th ICPEAC, Berlin 1983 , p. 601 .

[3] H. Schmidt, M.F. Vernon, P.S. Weiss, M.H. Covinsky, Y.T. Lee, XItI th ICPEAC, Berlin 1983, p. 642.

[4] T. TrickI and J. Wanner, J. Chem. Phys. 78, 6091 (1983)

[5] C. Crépin, J.L. Picqué, G. Rahmat, J. Vergès, R. Vetter and F.X. Gadea, M. Pélissier, F. Spiegelmann, J.P. Malrieu, $10^{\mathrm{e}}$ Colloque sur la Physique des Collisions atomiques et électroniques, Aussois (France) 1984.

[6] J. Weiner, J. Boulner, J. Keller and R. Bonanno, XIII th ICPEAC, Berlin 1983, p. 327 .

[7] A. Pesnelle, S. Runge and G. Watel, XIII th ICPEAC, Berlin 1983, p. 325.

[8] H. Haberland, Y.T. Lee and P.E. Siska, Adv. Chem. Phys. 47, 487 (1981). 
[9] Ch. Bender, W. Beyer, H. Haberland, D. Hausamann and H.P. Ludescher, this issue.

[10] W. Beyer, H. Haberland, D. Hausamann, XIII th ICPEAC, BerIIn, p. 344.

[11] V. Bocvarski, J. Robert, I. Colomb de Daunant, J. Reinhardt, J. Baudon, M. Dumont and $\mathrm{Ch}$. Lerminiaux, $10^{\mathrm{e}}$ Colloque sur la Physique des Collisions atomiques et êlectroniques, Aussois (France) 1984, p. 98.

[12] M. Dumont, J. Physique Lett. 41, L 275 (1980).

[13] J.M. Mestdagh, J. Pascale, XIII th ICPEAC, Berlin 1983, P. 341. J.M. Mestdagh, J. Berlande, P. de Pujo, J. Cuvellier, A. Binet, Z. Phys. A, 304,3 (1982).

[14] R. Düren, E. Hasselbrink and H. Tischer, XIII th ICPEAC, Ber1in 1983, p. 343.

[15] G. Jamieson, W. Reiland, C.P. Schulz, H.U. Tittes and I.V. Hertel, XIII th ICPEAC, Berlin 1983, p. 600.

[16] P. Pillet, R. Kachru, N.H. Tran, W.W. Smith and T.F. Gallagher, XIII th ICPEAC, Berlin 1983, p. 698 . 